\title{
El dibujo y su aporte a la identificación de valores de la arquitectura vernácula.
}

\author{
The contribution of drawings to the cultural heritage values \\ identification
}

Resumen

Julia Tamayo* jtamayoabril@gmail.com

Genoveva Malo** gmalot@uazuay.edu.ec Gabriela García*** kpuligv@hotmail.com

* Universidad de Cuenca ** Universidad del Azuay *** Universidad de Cuenca

Ecuador

Recibido:4/Abr/2018 Aceptado: 26/Ene/2019

a arquitectura vernácula es un legado cultural cuyo estudio ha ganado im-
portantes espacios en las últimas décadas. En ella destaca la fuerte pre-
sencia de saberes ancestrales que resultan en ejemplos de sencillez cons-
tructiva, máximo aprovechamiento de materiales locales e integración al paisaje.
En efecto, se trata de un patrimonio complejo y dinámico que exige un profundo
entendimiento para garantizar su conservación y uso como fuente de innovación
en la arquitectura contemporánea. En este estudio, se reflexiona sobre la impor-
tancia de la expresión gráfica como un instrumento generador y transmisor de
información, el cual favorece a una mayor comprensión de la arquitectura verná-
cula con respecto a sus valores culturales. El análisis se sustenta en teorías de la
imagen y en los datos cuanti-cualitativos obtenidos de la investigación e informa-
ción levantada en 1977-1978 de Arquitectura Popular de Azuay y Cañar. Entre los
aportes se evidencia el potencial de este recurso como lenguaje visual y de mayor
alcance en cuanto a su audiencia y ámbito de investigación.
Palabras clave: expresión gráfica, dato cualitativo, valoración.
Abstract:
Vernacular architecture is considered as a cultural legacy which study has gai-
ned prominence during the last decades. Indeed, its complex and dynamic na-
ture requires a depth understanding in order to guarantee its conservation and
to maximize its use as a source of innovation for contemporary architecture. This
paper reflects on the importance of drawings as a potential tool to communicate
and evidence the cultural heritage values attached to these modest buildings. A
comparative analysis is presented, contrasting quantitative data and qualitative
data obtained from the research carried out at the end of the eighties. The main
findings emphasize the role of drawings as a no verbal - visual language easy to
comprehend for diverse audiences and capable to communicate valuable infor-
mation to different fields.
Keywords: manual drawing, qualitative information, valorization process.

arquitectura vernácula es un legado cultural cuyo estudio ha ganado importantes espacios en las últimas décadas. En ella destaca la fuerte pretructiva, maximo aprovechamiento de materiales locales e integracion a paisaje. En efecto, se trata de un patrimonio complejo y dinámico que exige un profundo tancia de la expresión gráfica como un instrumento generador y transmisor de imagen y en los datos cuanti-cualitativos obtenidos de la investigación e información levantada en 1977-1978 de Arquitectura Popular de Azuay y Cañar. Entre los aportes se evidencia el potencial de este recurso como lenguaje visual y de mayor

Palabras clave: expresión gráfica, dato cualitativo, valoración.

\section{Abstract:}

Vernacular architecture is considered as a cultural legacy which study has gaiture requires a depth understanding in order to guarantee its conservation and to maximize its use as a source of innovation for contemporary architecture. This paper reflects on the importance of drawings as a potential tool to communicate comparative analysis is presented, contrasting quantitative data and qualitative data obtained from the research carried out at the end of the eighties. The main findings emphasize the role of drawings as a no verbal - visual language easy to comprehend for diverse audiences and capable to communicate valuable inforKeywords: manual drawing, qualitative information, valorization process. 


\section{Introducción}

El estudio de la arquitectura vernácula a lo largo de más de cuatro décadas, ha contribuido progresivamente a mejorar el entendimiento de su compleja y dinámica naturaleza, así como respecto a la importancia de su protección y conservación para las actuales y futuras generaciones. El presente trabajo constituye un aporte en este ámbito y pone en relieve el rol del dibujo como elemento generador, transmisor de conocimiento y como dato histórico que conlleva una comunicación cerebral, emotiva, que permite la interpretación del vivir y su entorno.

Las reflexiones planteadas en este texto basan su posicionamiento en entradas teóricas que, desde conceptos y teorías sobre la arquitectura vernácula y su valoración, así como particulares enfoques sobre el dibujo y la imagen, pretenden poner en valor el dibujo como recurso fundamental de conocimiento y de construcción de discursos. A través de la comparación de los resultados obtenidos de un análisis cuantitativo realizado para este tipo de estudios, que se complementa con una investigación cualitativa de interpretación y dialogo entre el dibujo y distintas miradas (interdisciplinaria), se pone en evidencia, para este caso, el enriquecimiento en la identificación de los valores de la arquitectura vernácula.

Los hallazgos principales de este ejercicio permiten afirmar que el dibujo cobra un nuevo sentido como lenguaje de la arquitectura para la interpretación de obras del pasado. El dibujo, como un modo de pensar y hacer, enfatiza su doble rol como herramienta para pensar al momento de la interpretación y su rol como herramienta de abstracción de una realidad (pensar para dibujar). Por otro lado, se pone de manifiesto que la representación de la arquitectura vernácula, mediante el dibujo, facilita el acceso a la información de manera más atractiva y didáctica a un público diverso. Además, la representación gráfica realizada a mano tiene más recepción que la fotografía o representación digital por su trazo más emotivo y expresivo.

\section{La arquitectura vernácula y su valoración}

En el transcurso del tiempo la arquitectura vernácula ha sido registrada, valorada y nominada desde varias miradas, antropológica, sociológica, tecnológica... Se la ha nombrado como: "autóctona (que ha nacido o se ha originado en el mismo lugar donde se encuentra), popular (perteneciente o relativo al pueblo), tradicional (que sigue las ideas, normas o costumbres del pasado)", (Tillería, 2010); y ha sido más bien una arquitectura identificada por su condición de pobreza, marginalidad y empirismo. A inicios del siglo XIX, el término vernáculo se utilizó para nombrar a los edificios 'típicos' de cada lugar presentes en las narrativas de los viajeros, misioneros u oficiales colonizadores de aquella época (Zorrilla, 2015). Ya en la década de los sesentas, expertos como Pablo Oliver en su obra 'Shelter and Society' y Amos Rapoport en su obra 'House Form and Culture', dejan en un segundo plano la noción de nostalgia, pobreza o subdesarrollo y centran el estudio de la arquitectura vernacular en los aspectos cultural, técnico y del entorno al concebirla como: "aquella en que no existen pretensiones teóricas o estéticas; que trabaja con el lugar de emplazamiento y con el microclima; respeta a las demás personas y sus casas y, en consecuencia, al ambiente total, natural o fabricado por el hombre, y trabaja dentro de un idioma con variaciones dentro de un orden dado" (Rapoport, 1969, p. 12 ).

El interés de tratar los temas vinculados con esta arquitectura se enfatiza a partir del movimiento moderno como una respuesta alternativa a los principios de la industrialización y el interés por los edificios de manufactura artesanal que recuerdan al movimiento inglés Arts \& Crafts. Desde entonces varios tratados $y_{1}$ sobre todo, el reconocimiento como un componente cultural por parte de los organismos internacionales, como la UNESCO y el ICOMOS, han hecho que se observe sus valores como un legado digno de preservarse y estudiarse; al respecto, Supic (1982) sostiene que la arquitectura vernácula, caracterizada por la simplicidad, ha sido fuente de inspiración para la construcción de las teorías propuestas por los arquitectos de la modernidad del siglo $X X$

El reconocimiento, de la arquitectura vernácula como patrimonio cultural de los pueblos, posibilita la implementación de medidas para su protección y salvaguarda establecidas por la Organización de las Naciones Unidas para la Educación, la Ciencia y la Cultura (UNESCO, 2003). Desde que los documentos <cartas> internacionales de patrimonio hablan del tema inmaterial y la importancia de que el patrimonio sea reconocido y valorado por los sectores anteriormente marginados, los valores patrimoniales adquieren otro significado, y se amplía el espectro de las personas y objetos que hacen y consumen bienes culturales; esto lo ratifica la Carta de Cracovia (2000) en donde se considera el patrimonio como un tema social-cultural, directamente ligado a la identidad y la memoria... "un monumento habla más allá de la materialidad que muestra, en él 'habla' lo que está presente pero también lo que está ausente." (Cirvini y Gómez, 2008).

Se trata de un habitar, más allá del hábitat o morada. Esta arquitectura definida dominantemente en su forma por el uso y los significados ligados a éste, es una institución creada para garantizar el desarrollo de la vida en plenitud en donde, según Iglesia (2011) convergen cuestiones como la construcción de la cultura e identidad. 


\section{El dibujo como generador y trasmisor de conocimiento}

Actualmente, la arquitectura vernácula es percibida como un producto cultural complejo, de carácter vivo y dinámico, resultado del diálogo con su entorno físico y humano: "se trata de un proceso continuo, que incluye cambios necesarios y una continua adaptación como respuesta a los requerimientos sociales y ambientales" (ICOMOS, 1999). Durante las últimas décadas, el ámbito que ha cobrado mayor interés, según la literatura internacional, se refiere al estudio de arquitectura vernácula y su aporte al desarrollo sustentable, el cua busca poner en balance soluciones económicamente viables, socialmente accesibles y ambientalmente amigables. En este sentido, la arquitectura vernácula ha establecido un diálogo equilibrado y responsable entre lo natural y lo construido, del cual podrían desprenderse lecciones para la arquitectura contemporánea, entendiendo las características particulares de cada lugar (Correia et al., 2014; ICOMOS, 2011).

A nivel nacional, y en cercana relación con el entendimiento internacional, la arquitectura vernácula es concebida como un producto cultural cargado de simbolismos y dinámica, pues "ha sabido incorporar nuevos conocimientos que han sido adaptados o fusionados por su comunidad de acuerdo con el medio ambiente y necesidades" (Pesántez y González, 2011, p. 16). En este contexto, la protección del patrimonio surge a partir de la creación de la Casa de la Cultura Ecuatoriana, en 1942. Sin embargo, es en la década de los setenta, tras una serie de eventos modernizadores que transformaron considerablemente las ciudades, cuando se realizan importantes esfuerzos por proteger el patrimonio cultural edificado. Este es el caso de proyecto: Arquitectura popular de las provincias del Azuay y del Cañar, financiado por el Centro Interamericano de Artesanías y Artes Populares (CIDAP), organismo creado en 1975 para impulsar la artesanía artífice y la cultura popular en el Ecuador.

Como resultado de esta investigación (1977-1978), se realizó un registro que incluye la identificación de 1003 ejemplos de arquitectura popular en 14 cantones y 48 parroquias de la provincia del Azuay, y a 7 cantones y 30 parroquias de la provincia del Cañar. En ambas provincias se identificaron ejemplos emplazados en entornos urbanos y rurales, ubicados en zonas climáticas diferentes, cuya altitud varía entre los $24 \mathrm{msnm}$ hasta los $3806 \mathrm{msnm}$ sobre el callejón interandino, al Sur del Ecuador. El registro contiene datos de ubicación, técnicos de materialidad, estado de conservación, plantas arquitectónicas, detalles constructivos, así como una importante documentación gráfica y fotográfica. Información que no se la procesó ni difundió hasta que fue retomado como proyecto de investigación por la Facultad de Arquitectura y Urbanismo de la Universidad de Cuenca para la XIII convocatoria DIUC (2015-2016), en colaboración de la Universidad del Azuay: "Arquitectura del Azuay y Cañar hasta los años 80" (AVAC).
La presente investigación parte del entendimiento del dibujo como un medio de generación y transferencia de conocimiento. Esta afirmación se sustenta en la teoría del acto de visualización como comprensión de fenómenos ocultos en el dibujo de Joan Costa (1998). De acuerdo con este autor, el poder del dibujo va más allá de la simple representación, “...existe otra realidad que no podemos ver, ni directa, ni indirectamente, por el sencillo hecho de que no es una realidad visual, sino un universo de fenómenos" (Costa, 1998, p. 13). Esta otra realidad, como la denomina Costa, y el conjunto de fenómenos, están plasmados en los dibujos a la espera de nuestra interpretación. El dibujo encierra un conjunto de hechos y relaciones que representan la vida misma, y que en este estudio hemos denominado los Valores de la Arquitectura Vernácula.

Recurrir al dibujo para conocer e interpretar lo profundo de la arquitectura vernácula es una actividad visual e intelectual que busca escudriñar más allá de lo visible, conceptos y valores que están plasmados en líneas, trazos y escenas, tal como lo indica Costa, "...el deseo de aprehender los fenómenos invisibles es tan fuerte como el deseo de hacerlos comunicables (...) tratar de comprender, y después explicar, tales fenómenos supone un trabajo de la mente y de la mano ayudadas por medios técnicos; un trabajo de traducción de lo real, de su plasmación por medios visuazles, o más exactamente, gráficos. Así, fenómenos invisibles devienen realidades visualizadas, esto es, hechas visibles" (1998, p. 14).

El dibujo se convierte así en un recurso para transferir conocimiento, un medio didáctico que es capaz de hacer visible y comprensible lo invisible y complejo. El dibujo es entendido como un lenguaje, un texto, capaz de acercarnos a la comprensión de realidades y relaciones multidimensionales "que nos permiten insertar un objeto de un anticuario en un contexto distinto, disfrutar de él por lo que entonces ya significaba, pero también utilizarlo por las connotaciones que podemos atribuirle con nuestros léxicos actuales. Se trata de una sucesión de sorpresas, de aventuras al descubrir en una forma sus contextos originales y crear otros nuevos." (Eco, 1976, p. 274); en este caso, conocer la esencia de la arquitectura vernácula, no su mera descripción, sino sus valores, a partir de la observación e interpretación de gráficos que trasmiten historia, técnica, cultura, entre otros aspectos.

\subsection{EI dibujo como discurso}

Santos Zunzunegui en su libro Pensar la imagen, aborda el tema de la enunciación visual como un acto del lenguaje capaz de producir un discurso. En sus palabras:

"cuando se habla de enunciación, se concibe como un acto de lenguaje, a través del cual una estructura referencial produce un discurso. Se concibe, por lo tanto, como pura instancia lingüística, lógicamente presupuesta por la misma 
existencia de un enunciado en el que aparecen, de manera más o menos explícita, una seria de marcas que producen una ilusión enunciativa capaz de remitir al enunciador efectivo" (Zunzunegui, 2007, p. 81)

El mismo autor reconoce la presencia de un juego conversacional, que se produce entre quien hace el dibujo y quien lo interpreta. Es decir, "Intenta comprender, hacer al caso individual significativo en el contexto de la teoría, provee nuevas perspectivas sobre lo que se conoce, describe, explica, elucida, construye y descubre" (Zunzunegui, 2007, p. 29).

En este sentido se enfatiza la capacidad de la expresión gráfica de construir realidades a manera de discurso desde una postura, casi como una enunciación. $E$ dibujo entendido como un lenguaje que articula signos y significados para construir relatos; éste relato, en el caso de la arquitectura vernácula, es capaz de ponerla también en valor, pues a través de la gráfica se ha propuesto un sistema de valores (García et. al, 2017), que cobran sentido a través de la interpretación de los dibujos.

El discurso que ciertamente construye la arquitectura vernácula es el semiótico, Humberto Eco, (1987) en su libro "La estructura ausente" propone comprender la arquitectura desde sus funciones primarias y secundarias, así, al trasladar estos conceptos a la arquitectura vernácula la entendemos como una forma primitiva y básica de habitar, por lo tanto, la función principal es la de utilitaria a la que le llamamos primaria o denotativa, sin embargo, esta función deriva en unos significados que los Ilamamos secundarios o connotativos. Así vemos que uno de los elementos comunes en la mayoría de las edificaciones vernáculas es el corredor o poyo, denota un espacio semi-publico, primario, que se atraviesa y en el que se está, y connota un espacio social, de convivencia, de recibimiento, por lo tanto, es quizás uno de los espacios más utilizados de la casa y también el más significativo, el de los encuentros con toda la familia, con los vecinos y ambiente.

\subsection{El dibujo como dato cualitativo}

A diferencia de los campos alfa-numéricos que forman parte de un registro, en este caso de la arquitectura vernácula, el dibujo plasma de manera minuciosa el momento de vida en el que se realizó la captura, así como escenas de donde se desprende el uso del espacio, actividades de los habitantes e interacción con territorio. A nivel arquitectónico, el trazo y textura de un detalle constructivo, permiten solventar dudas y aclarar relaciones en el uso e interacción de estos materiales, para finalmente poner a la luz ese saber ancestral oculto en un proceso de ensayo-error permanente.

Se puede inferir entonces que de acuerdo con lo establecido por Vasilachis (2006, p. 29), la lectura crítica o ejercicio interpretativo, "intenta comprender, hacer al caso individual significativo en el contexto de la teoría, provee nuevas perspectivas sobre lo que se conoce, describe, explica, elucida, construye y descubre". Por lo cual, el dibujo forma parte de los datos, como un dato cualitativo, cuyo análisis, merecen una lectura reflexiva e interpretativa de cada imagen y en cada contexto, para aproximarnos a las particularidades temporales de una edificación y su entorno, y al mismo tiempo construir una reflexión epistemológica que dé cuenta de la realidad de la arquitectura vernácula.

Demostrar visualmente los valores y atributos de la arquitectura vernácula significa revalorar unas formas de habitar pretéritas, "esto quiere decir que el objeto no va a ser más víctima del olvido, y el consumo, no va a ser protagonista pasivo de la recuperación, sino que será el estímulo, la comunicación de operaciones posibles, capaces de adecuarlo continuamente a unas situaciones variables en el curso de la historia; operaciones que habrán de ser actos de decisión responsable, de valoración ajustada de las formas, de sus elementos constitutivos, de las configuraciones que pueden asumir, y por ello, de las bases ideológicas que las han de justificar"(Eco, 1967, p. 277).

\section{Materiales y métodos}

Este artículo complementa los resultados presentados en un artículo anterior titulado "Valoración de la arquitectura vernácula de Azuay y Cañar" (VAVAC), elaborado por los mismos autores (García, Tamayo y Malo, 2017). En este caso, se hace énfasis en la teoría que sustenta la documentación gráfica como elemento generador y transmisor de información para el entendimiento de realidades complejas, tales como las de la arquitectura vernácula.

A partir de los resultados alcanzados en la investigación anterior, se desarrolla una reflexión comparativa entre las variables cuantitativas tales como emplazamiento, entorno, número de pisos y otros, que fueron analizadas a través de programas estadísticos, y la información gráfica visual de los dibujos realizados a tinta y a mano alzada, por parte de los dibujantes de los años setenta, así como registros actualizados al 2017 realizados por parte de los autores. Con estos insumos se realizó una lectura crítica, reflexionando e interpretando cada imagen para descubrir las características que relaten con mayor veracidad este tipo de arquitectura.

Indudablemente el estudio se inscribe en el paradigma interpretativo que navega entre la interpretación y comprensión de esas imágenes, de la vida cotidiana y las preguntas y respuestas que surgen de estas. Según Vasilachis:

"el paradigma interpretativo está basado en teorías como el interaccionismo simbólico, la fenomenología, la hermenéutica, la etnometodología (...) Las que señalan la importancia de estudiar la acción y el mundo social desde el punto de vista de los actores. La investigación cualitativa se apoya y depende de una concepción orientada hacia el significado, el contexto, la interpretación la comprensión y la reflexividad" (2006, p. 50). 
Las imágenes de la arquitectura vernácula, de la investigación "Arquitectura vernácula del Azuay y Cañar hasta los años 80", como dato cualitativo, se analizan desde la semiología, en consideración, como ya lo sustentamos, de que la arquitectura, es un producto de la cultura y ésta, a su vez, un sistema de signos que comunican (Eco, 1986). Los dibujos son los elementos que significan y expresan una información que trataremos de organizar e interpretar mediante un sistema de cinco valores planteado en el artículo VAVAC (García et. al, 2017):

1) Técnico, referido al saber hacer o know-how, probado de manera empírica (ensayo-error) a lo largo del tiempo. Expresa la significación de los ingeniosos modos de solucionar aspectos constructivos y estéticos de los materiales, así como de los procesos que caracterizan y definen una construcción, los cuales han persistido por su efectividad y eficiencia en el aprovechamiento de los recursos locales.

2) Habitabilidad, es la noción de construcción de sentido del uso y función de un espacio; es un concepto con un gran nivel de abstracción, ya que involucra satisfacer las necesidades básicas, las espirituales, las sociales, cosmológicas, culturales y más. En consecuencia, es el valor que le confiere significado a la edificación.

3) Estético, referido a aspectos como armonía, y equilibrio entre la forma construida, que representa lo útil y bello. Es la noción de construcción de sentido de la forma, que en su contemplación provoca emociones y experiencias dominantemente vinculadas a la condición de belleza.

4) Ambiental, su significación radica en el respeto e integración con el entorno natural, contexto paisajístico y uso de materiales y procesos locales.

5) Económico, se refiere a saber utilizar lo suficiente, ni menos ni más, es decir presenta una alta eficiencia energética. Es un valor asociado a la coherencia y optimización en el de uso en espacios, maximización y priorización del uso de materiales del entorno inmediato, lo cual favorece la construcción in-situ que reduce la huella ecológica y abarata costos de transporte, y facilita su mantenimiento constante.

Estos valores se encuentran íntimamente relacionados entre sí a través de seis atributos principales o fuentes de verificación que objetivizan lo abstracto del valor, como son:

- Uso-función: dominantemente vivienda vinculada a actividades agrícolas;

- Técnica constructiva: dominantemente en tierra tales como adobe, bahareque, piedra, seguidas de madera y caña;

- Morfología: referida a aspectos de la forma y diseño tales como geometría, proporciones, escala, relaciones lleno-vacío, etc.

- Emplazamiento: referido dominantemente a aspectos de relieve o topografía; $y$ finalmente

- Materiales locales empleados en la construcción y ornamentación de la arquitectura vernácula.

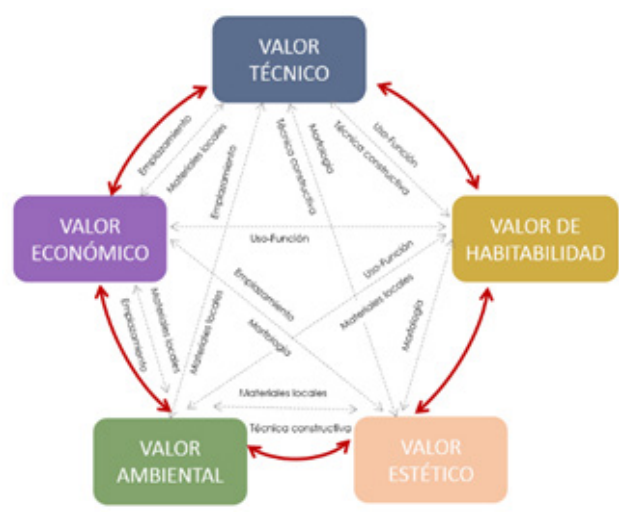

Ilustración 1: Esquema de valores e interrelaciones a través de atributos.

Fuente: García et al. (2017). VAVAC.

\section{Análisis de los valores de la arquitectura vernácula a través del dibujo}

A la luz de las teorías antes expuestas, se comprende por valor a los significados de unos significantes que denominamos atributos; éstos son complementarios y consecuentes unos de otros. Así, los dibujos de esta arquitectura no solamente muestran un significante, un objeto para vivir, sino que además exteriorizan unos significados tan importantes que la convierten en un objeto que comunica y enseña unos significados del habitar, del ser.

Mediante una lectura crítica interpretativa se leen los dibujos como dato cualitativo, portador de sentido; se analiza en cada uno de los casos y se compara con los datos del análisis cuantitativo, contrastando las variables o atributos con la lectura crítica interpretativa de los dibujos, para tener como resultado las características de los valores.

Para comprender el aporte del dibujo a la determinación de estos resultados, es necesario referirse a las variables alfanuméricas de las fichas de registro elaboradas como parte de la investigación CIDAP (1977-1978), que se sintetizan en la tabla 1.

Para mayor detalle en el análisis, se parte de cada variable, dato cuantitativo, para luego de la interpretación crítica de los dibujos complementar la información y llegar a definir los valores. Cabe aclarar, como ya se hizo anteriormente en la definición de valores, que éstos no son únicos ni totales: más bien se relacionan y complementan mediante los atributos que los vinculan. Así, un dibujo confirma los datos cuantitativos, pero a la vez muestra otros atributos tal vez los más significativos que enriquecen la lectura, y por lo tanto multiplica los valores. Esto es justamente lo que demuestra la riqueza de la arquitectura vernácula. 


\begin{tabular}{|c|c|c|}
\hline VARIABLES & $\begin{array}{l}\text { Cant. } \\
\mathrm{N}=1003\end{array}$ & $\begin{array}{c}\text { Porcen } \\
\%\end{array}$ \\
\hline \multicolumn{3}{|l|}{ EMPLAZAMIENTO } \\
\hline Aislada & 445 & 44,37 \\
\hline Continua con portal & 119 & 11,86 \\
\hline Continua con retiro frontal & 2 & 0,20 \\
\hline Continua sin retiro frontal & 143 & 14,26 \\
\hline Pareada con retiro frontal & 1 & 0,10 \\
\hline Pareada sin retiro frontal & 291 & 29,01 \\
\hline Vacías / No registrado & 2 & 0,20 \\
\hline \multicolumn{3}{|l|}{ ENTORNO } \\
\hline Agrícola & 493 & 49,15 \\
\hline Edificado & 509 & 50,75 \\
\hline No registrado & 1 & 0,10 \\
\hline \multicolumn{3}{|l|}{ NUM PISOS } \\
\hline 1 Piso & 370 & 36,89 \\
\hline 2 Piso & 588 & 58,62 \\
\hline 3 Piso & 42 & 4,19 \\
\hline 4 Piso & 2 & 0,20 \\
\hline 5 Piso & 1 & 0,10 \\
\hline \multicolumn{3}{|l|}{ CIMIENTOS } \\
\hline Caña & 2 & 0,20 \\
\hline Concreto & 1 & 0,10 \\
\hline Ladrillo & 2 & 0,20 \\
\hline Madera & 8 & 0,80 \\
\hline Mármol & 1 & 0,10 \\
\hline Piedra & 967 & 96,40 \\
\hline Pilotes & 6 & 0,60 \\
\hline Vacías / Ninguno & 16 & 1,60 \\
\hline \multicolumn{3}{|l|}{ SOPORTES - COLUMNAS } \\
\hline Madera & 911 & 90,83 \\
\hline Otros & 92 & 9,17 \\
\hline \multicolumn{3}{|l|}{ VIGAS } \\
\hline Madera & 962 & 95,91 \\
\hline Otros & 41 & 4,09 \\
\hline \multicolumn{3}{|l|}{ MUROS } \\
\hline Adobe & 464 & 46,26 \\
\hline Bahareque & 403 & 40,18 \\
\hline Bloque & 5 & 0,50 \\
\hline Caña & 23 & 2,29 \\
\hline Ladrillo & 21 & 2,09 \\
\hline Madera & 21 & 2,09 \\
\hline Piedra & 45 & 4,49 \\
\hline Tapial & 14 & 1,40 \\
\hline Vacías / Ninguno & 7 & 0,70 \\
\hline
\end{tabular}

\begin{tabular}{|c|c|c|}
\hline CUBIERTA & & \\
\hline Asbesto & 1 & \\
\hline Bijao & 1 & \\
\hline Ladrillo & 1 & \\
\hline Hoja & 6 & \\
\hline Teja & 855 & \\
\hline Paja & 62 & \\
\hline Zinc & 71 & \\
\hline Vacías / No registrado & 6 & \\
\hline PISOS & & \\
\hline Baldosa & 5 & 0,50 \\
\hline Caña & 1 & 0,10 \\
\hline Cemento & 76 & 7,58 \\
\hline Ladrillo & 125 & 12,46 \\
\hline Madera & 140 & 13,96 \\
\hline Piedra & 44 & 4,39 \\
\hline Tierra & 609 & 60,71 \\
\hline Vacías / No registrado & 3 & 0,30 \\
\hline CARPINTERIA PUERTAS & & \\
\hline Madera & 842 & 83,95 \\
\hline Madera y vidrio & 3 & 0,30 \\
\hline Madera hierro & 1 & 0,10 \\
\hline Caña guadua & 3 & 0,30 \\
\hline Vacías / No registrado & 154 & 15,35 \\
\hline CARPINTERIA VENTANAS & & \\
\hline Madera & 631 & 62,91 \\
\hline Madera y vidrio & 93 & 9,27 \\
\hline Madera y hierro & 5 & 0,5 \\
\hline Caña guadua & 3 & 0,3 \\
\hline Zinc & 1 & 0,1 \\
\hline Otros & 1 & 0,1 \\
\hline Solo abertura & 5 & 0,5 \\
\hline Vidrio & 2 & 0,2 \\
\hline $\begin{array}{c}\text { Vacías / No registrado / } \\
\text { Ninguno }\end{array}$ & 262 & 26,12 \\
\hline
\end{tabular}

Tabla 1: Características de las edificaciones vernáculas de Azuay y Cañar a partir de sus principales componentes. Fuente: AVAC (2017).

\subsection{Emplazamiento}

Mientras los resultados alfa numéricos indican que el tipo de emplazamiento dominante en estas provincias corresponde a vivienda aislada, el dibujo nos permite observar algunos elementos que le dan valor al tipo de emplazamiento, y por ende definen el "carácter y valor de habitabilidad". Por ejemplo, el aprovechamiento del 
perfil topográfico irregular o con significativos desniveles, propios de la región montañosa sobre la que se emplazan, con el fin de conformar espacios complementarios a la vivienda, generalmente vinculados a actividades agrícolas, agropecuarias y lúdicas (figura 1,2,3 y 4).

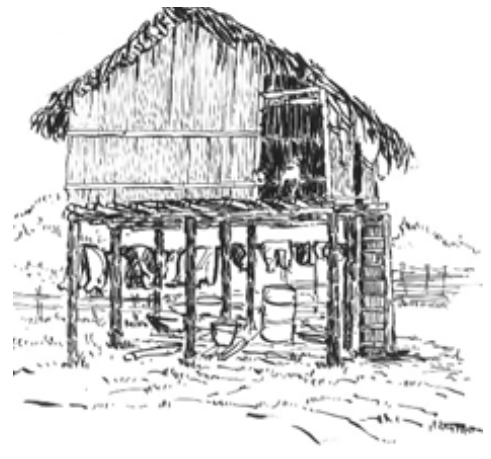

Figura 1 VALOR AMBIENTAL/TÉCNICO. Lugar mínimo y suficiente. Convivencia con la naturaleza, vegetal y animal. Aprovechamiento técnico de la estructura para multiusos (Cañar, Pancho Negro).

Fuente: AVAC. (2017).

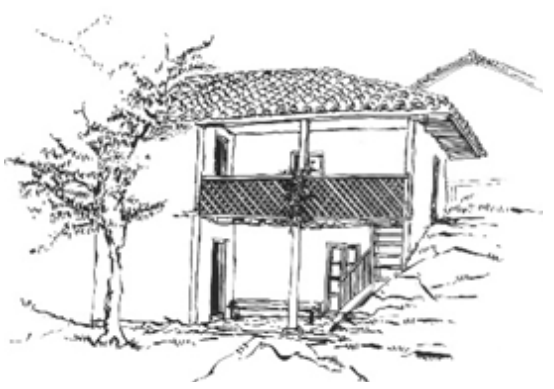

Figura 2: VALOR ECONOMICO/ESTÉTICO. Integración armoniosa del emplazamiento a desnivel, con una fuerte expresividad de espacios semipúblicos que denota una función social. (Cañar, Taday).

Fuente: AVAC (2017).

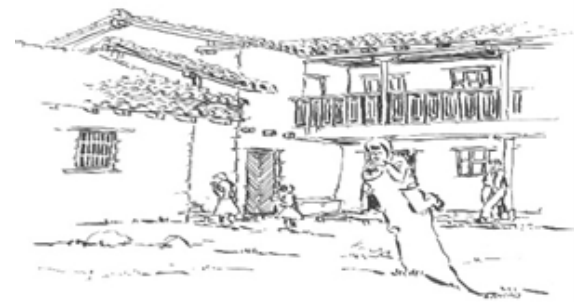

Figura 3: VALOR HABITABILIDAD. Edificaciones continuas que integran exterior-interior, con el uso para juegos y descanso. Espacio que denota confianza y tranquilidad (Azuay, El Progreso).

Fuente: AVAC (2017).

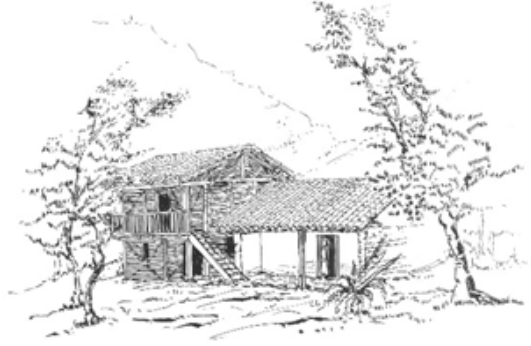

Figura 4: VALOR TÉCNICO. Viviendas pareadas Volúmenes similares contactados lateralmente, que se contraponen en altura y textura. Sin embargo, se integran con el entorno y elementos constructivos compartidos (Cañar, Sageo)

Fuente: AVAC (2017).

\subsection{Entorno}

En relación al entorno, los datos alfanuméricos indican un entorno dominantemente edificado o urbano, sobre un entorno agrícola. Sin embargo, el dibujo nos permite identificar las formas de habitar estos territorios, las cuales son identificadas por la presencia de los objetos en los dibujos, así como la representación humana. Así, por ejemplo, en el mundo urbano se identifican los balcones como los espacios "sociales" que permiten la relación con el otro, o tiendas en las plantas bajas que conviven con el uso residencial en las plantas superiores. Al contrario, el entorno agrícola, muestra formas de convivencia cercana con actividades agrícolas y de crianza de animales, donde los portales son el lugar de trabajo, donde se alberga la cosecha y en muchos de ellos se trabajan artesanías (figuras $5,6,7$ y 8 ).

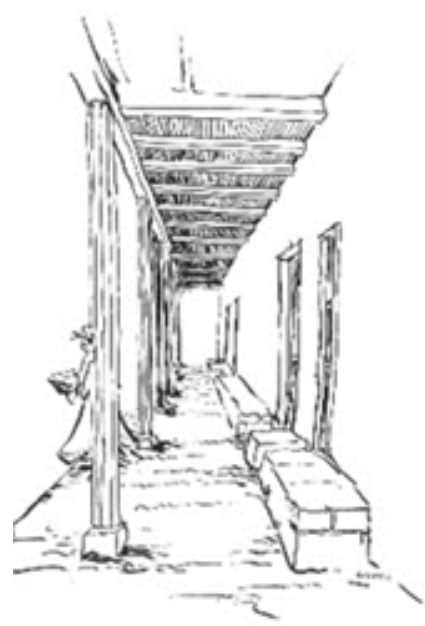

Figura 5: VALOR TÉCNICO/HABITABILIDAD. Espacio semi-público, de reunión y trabajo con fuerte presencia de la técnica artesana en los materiales naturales que confieren calidez y propiedad (Azuay, Santa Ana). Fuente: AVAC (2017). 


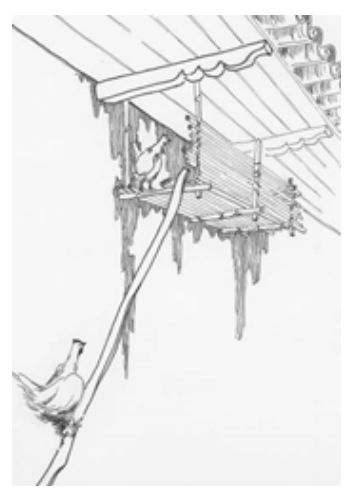

Figura 6: VALOR ESTÉTICO/AMBIENTAL. Uso y función. Detalle de aprovechamiento de un alero para alberge de aves domésticas que propician el sustento y muchas veces compañía (Jadán, Azuay).

Fuente: AVAC. (2017)

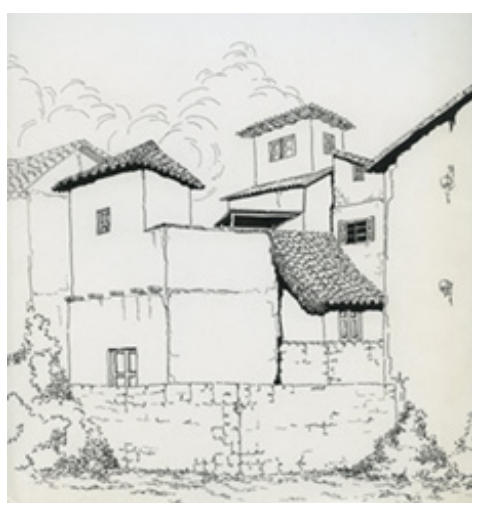

Figura 7: VALOR HABITABILIDAD/ESTÉTICO. Conjunto armonioso y singular de casas adosadas en un entorno urbano. Evidencian la técnica constructiva en tierra y piedra (Ingapirca, Cañar).

Fuente: AVAC. (2017)

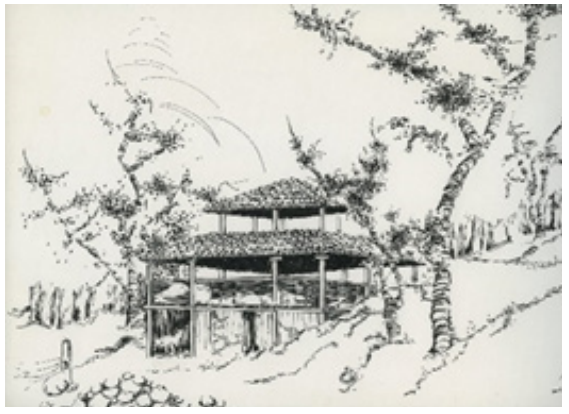

Figura 8: VALOR TÉCNICO/ECONÓMICO. Edificación de formas ligeras y transparentes para elaboración de ladrillos adaptada a un entorno agrícola y a desnivel. Estructura constructiva en madera y bahareque (Cuenca, Azuay).

Fuente: AVAC (2017).

\subsection{Número de pisos}

Si bien los datos referidos al número de pisos permiten tener una estimación de la altura de la edificación, el dibujo pone en evidencia la fragilidad de este dato donde una edificación con un determinado número de pisos, por ejemplo 2, no mantiene la misma altura que otra con el mismo número de pisos (fig. 10). Es además importante resaltar que el valor de la morfología no está determinado por un análisis en dos dimensiones, sino en tres y hasta cuatro dimensiones (incluyendo el tiempo y las transformaciones que ha sufrido), dimensiones que en este caso han sido valoradas y apreciadas desde el dibujo (figuras 9, 10, 11 y 12).

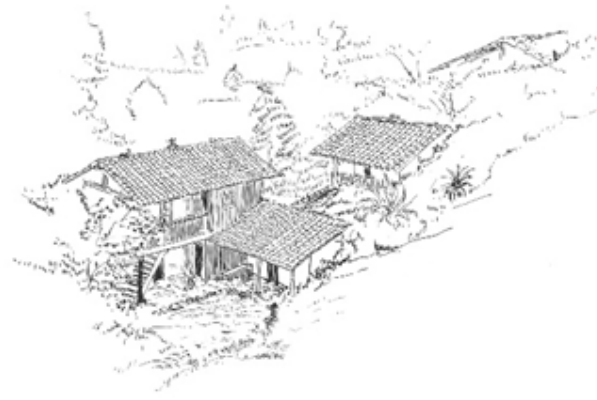

Figura 9: VALOR AMBIENTAL/ESTÉTICO. Emplazamiento de fuerte acoplamiento con el desnivel del terreno, el paisaje y los niveles de edificación (Azuay, Sidcay). Fuente: AVAC (2017)

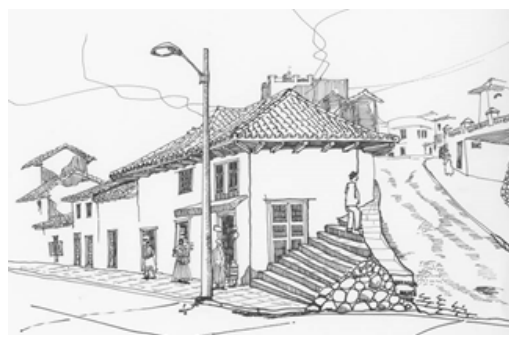

Figura 10: VALOR HABITABILIDAD/AMBIENTAL. Casa de dos pisos que aprovecha el desnivel para ganar altura. Morfología regular y armónica del tramo con el entorno edificado (Cuenca, Azuay).

Fuente: AVAC (2017).

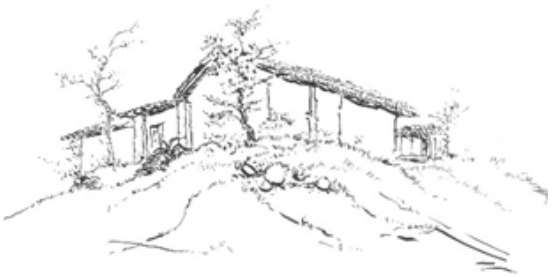

Figura 11: VALOR AMBIENTAL/ECONÓMICO. Visuales que magnifican la altura de una edificación vernácula por la simbiosis con la morfología del terreno (Azuay, Llacao). Fuente: Fuente: AVAC. (2017). 


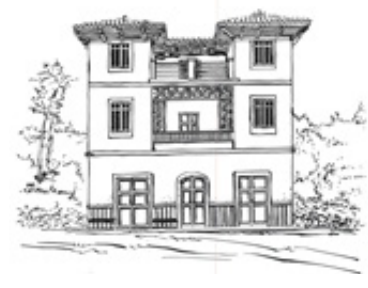

Figura 12: VALOR HABITABILIDAD/TÉCNICO. Casa de tres pisos con clara simetría vertical, con terrazas centrales y detallado trabajo de carpintería (Cañar).

Fuente: AVAC (2017).

\subsection{Cimentación y sobrecimientos}

De la misma manera que en los casos anteriores, si bien el análisis cuantitativo permite identificar el materia dominante utilizado en este componente constructivo, en este caso piedra, no permite valorar la excepcionalidad de la arquitectura vernácula ni la creatividad de sus constructores como lo hace el dibujo. A través de este recurso, se identifica un valor tanto ambiental como estético, al trabajar con los materiales que se encuentran insitu e incorporarlos para que cumplan la función de soporte de la edificación en la forma de cimientos, sobrecimientos o basas de pilares, en el caso de estructuras de madera, las cuales a su vez presentan elaborados diseños y formas (figuras 13 y 14).

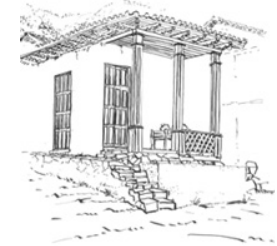

Figura 13: VALOR ECONÓMICO/ TÉCNICO. Técnica constructiva de sobrecimiento de piedra, que nivela el terreno para ganar espacio para el corredor y conferirle simpleza y funcionalidad al espacio (Cañar, Biblián). Fuente: AVAC (2017).

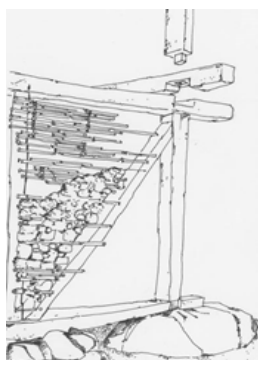

Figura 14: VALOR TÉCNICO/AMBIENTAL. Estructura de bahareque. Ingenioso sistema de ensamble y apoyo en elementos pre existente en el territorio, como piedra, basa y aprovechamiento para cámara de aire (Azuay, Paute)

Fuente: AVAC (2017)

\subsection{Columnas y vigas}

Los elementos estructurales de la edificación vernácula de las provincias del Azuay y Cañar, responden dominantemente a una materialidad de madera. Nuevamente, este dato se limita a una descripción genera que es ampliamente enriquecida con el análisis del dibujo, de donde se desprenden los valores: ambiental, económico, estético, técnico. En las ilustraciones podemos evidenciar incluso la temporalidad: mientras la columna "panzona" es un rezago de la colonia, la cuadrangular es actual (figura 15).
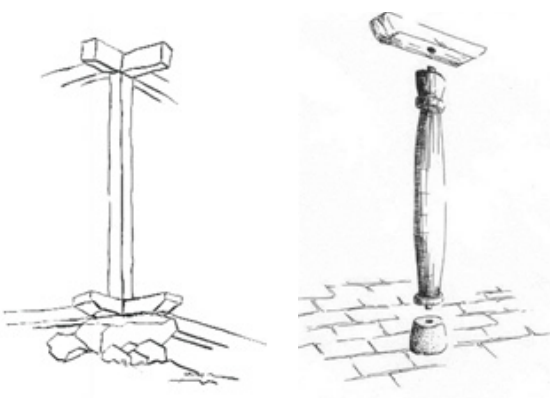

Figura 15: Columna rústica de madera, materiales locales, (tal vez producto de una carpintería de ribera) sobre piedra que hace las veces de basa (Cuenca, Azuay). Columna tallada, "panzona", con basa de piedra y zapata. Materiales locales (Cuenca, Azuay). Fuente: AVAC. (2017)

\subsection{Muros}

Los datos alfanuméricos permiten determinar la materialidad dominante de este tipo de arquitectura, que corresponde a edificaciones en tierra, pero el dibujo permite adscribir a esta materialidad un valor estético que surge como resultado de la técnica constructiva, como en el caso de los muros de piedra o caña, o de la ausencia de revoque, en el caso de los sistemas constructivos de adobe, o a su vez de la expresión alcanzada por la edificación como resultado de la combinación de materiales. Al mismo tiempo, el dibujo ha permitido observar criterios constructivos de adaptabilidad y agregación, los cuales confieren unidad, donde el principio de continuidad horizontal y vertical del sistema estructural, principalmente en los sistemas constructivos de bahareque, es observado en uniones y riostras, utilizando la cruz de San Andrés como figura triangular menos deformable (figuras $17,18,19$ y 20). 


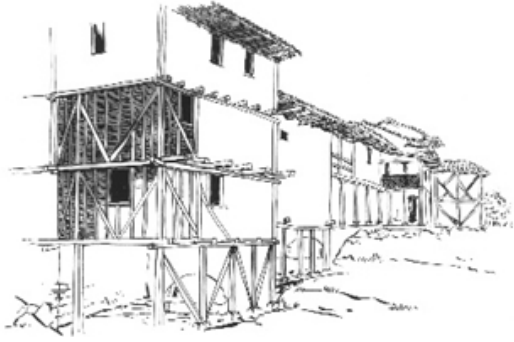

Figura 16: VALOR TÉCNICO/ AMBIENTAL. Estructura de bahareque adaptable al desnivel del terreno, que muestra con claridad el esqueleto de madera, que se caracteriza por su ligereza y absorción de esfuerzos horizontales: por lo tanto, es una estructura antisísmica. (Santa Ana, Azuay).

Fuente: AVAC (2017).

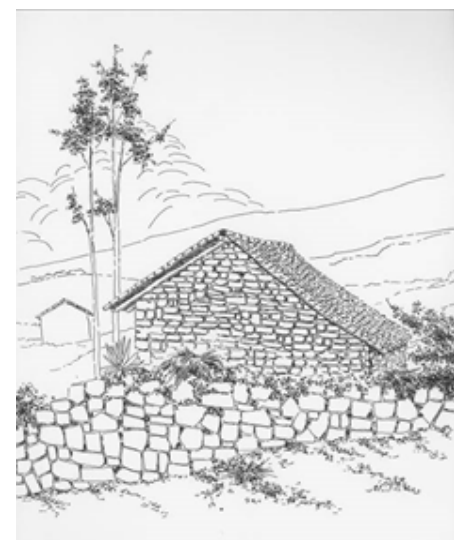

Figura 17: VALOR AMBIENTAL/TÉCNICO. Materiales locales. La piedra es utilizada en los muros portantes de la edificación y en el muro de cerramiento, expresando una completa simbiosis con el entorno (Bayas, Cañar). Fuente: AVAC (2017).

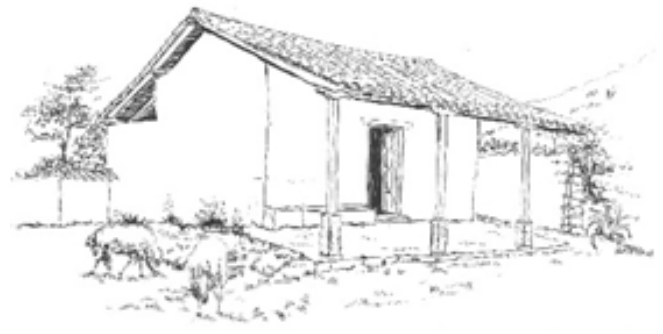

Figura 18: VALOR ECONÓMICO/HABITABILIDAD Edificación típica de morfología simple y sobria, en tierra, madera y teja, con corredor delantero y habitación, que demuestra total funcionalidad (San Juan, Azuay). Fuente: AVAC (2017)

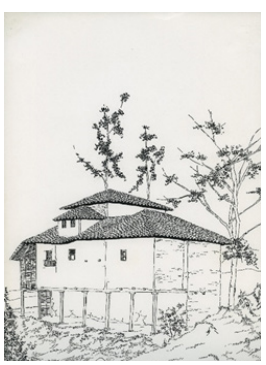

Figura 19: VALOR ESTÉTICO/TÉCNICO. El bahareque, por ligero y resistente, posibilita varios niveles de edificación, al igual que permite juegos de volúmenes lleno-vacíos y escalas (Santa Ana, Azuay).

Fuente: AVAC (2017).

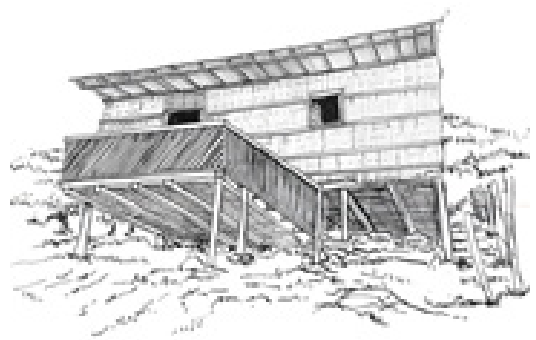

Figura 20: VALOR AMBIENTAL/TÉCNICO. Materiales locales, caña guadua como estructura y muros, que confieren levedad y naturalidad a una edificación de la costa (Cañar)

Fuente: AVAC (2017)

\subsection{Cubiertas}

Respecto a las cubiertas, si bien la mayoría son de teja, todavía quedan las de paja que confieren un aspecto rústico-tradicional y son muy funcionales en los climas fríos del páramo. La del dibujo (fig. 23) ha permitido la identificación de valores simbólicos expresados en elementos de ornamentación que se colocan sobre la cubierta (cruz) y en torno a los cuales orbita una de las festividades más importantes en la vida de la comunidad donde se inserta la edificación (el enteche). (figuras 21, $22,23$ y 24$)$

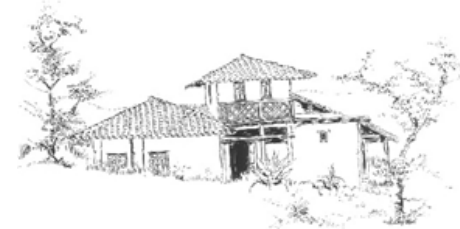

Figura 21: VALOR AMBIENTAL/TÉCNICO. Armonía de volúmenes en que se conjuga las cubiertas con e entorno natural y desnivel del terreno. Juego pintoresco de escalas e inclinación de cubiertas (Santa Ana, Azuay). Fuente: AVAC (2017) 


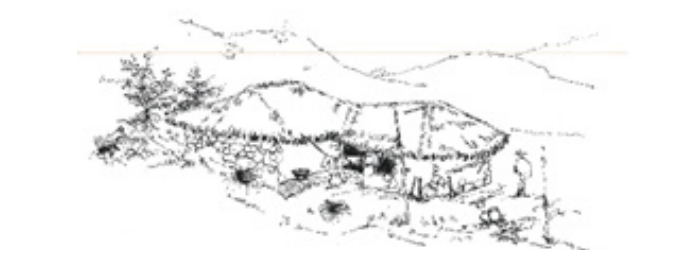

Figura 22: VALOR AMBIENTAL/HABITABILIDAD. Materiales locales, cubiertas con la típica paja de cerro, que remata los gruesos muros de piedra para cobijo del clima frío de las montañas andinas (Juncal, Cañar). Fuente: AVAC (2017).

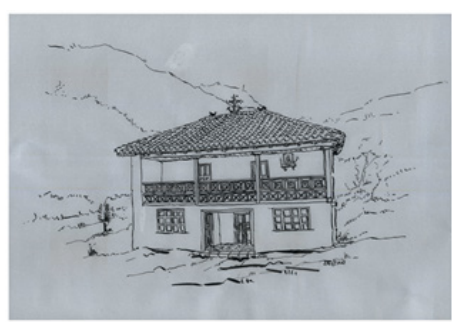

Figura 23: VALOR HABITABILIDAD/AMBIENTAL. Cubierta de teja a cuatro aguas con la típica cruz y corredor superior con imágenes religiosas que identifican la religiosidad de sus habitantes (Santa Ana, Azuay). Fuente: AVAC (2017)

\subsection{Pisos}

La tabla 1 indica una mayor presencia de pisos de tierra, siendo los pisos de piedra los de menor importancia cuantitativa. Sin embargo, el dibujo contribuye a revertir radicalmente esta importancia, pues da cuenta de unos ingeniosos modos de conseguir aportes estéticos con la combinación de otros materiales, tales como huesos de animales, en edificaciones de entornos urbanos dominantemente, marcando un estatus frente a sus análogas en los contextos rurales (figuras 24 y 25).

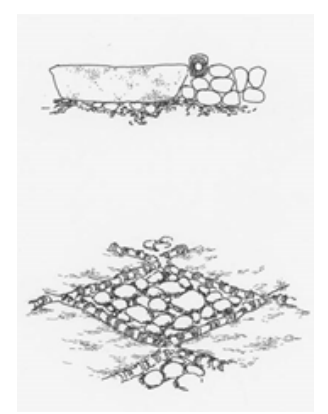

Figura 24: VALOR ESTÉTICO/TÉCNICO. Revestimiento de piso, conocido como enmorrillado de piedra con diseño geométrico y uso de huesos de animales como elementos de ornamentación (Azogues, Cañar). Fuente: AVAC (2017).

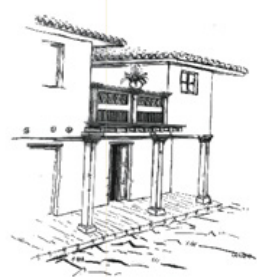

Figura 25: VALOR ECONÓMICO/TÉCNICO. Piso de ladrillo de una vivienda urbana, que posibilita buen mantenimiento y expresividad (Cañar).

Fuente: AVAC (2017).

\subsection{Carpinterías}

De manera similar a los elementos estructurales, columnas y vigas, el dibujo ha permitido poner en evidencia la riqueza de variadas formas presentes en las edificaciones vernáculas, tanto en entornos urbanos como rurales. Estos ricos y variados diseños confieren una característica de excepcionalidad a cada edificación y muchos de ellos son la interpretación y representación de una cosmovisión del mundo materializada en los elementos básicos de una edificación (figuras 26 y 27).

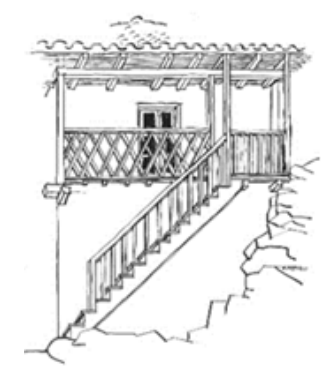

Figura 26: VALOR TÉCNICO/ECONÓMICO. Arte y oficio del trabajo de estructura y carpintería en madera. Diseño, modulación e integración de enrejados, escaleras y estructura de en dos plantas (Rivera, Cañar).

Fuente: AVAC (2017).

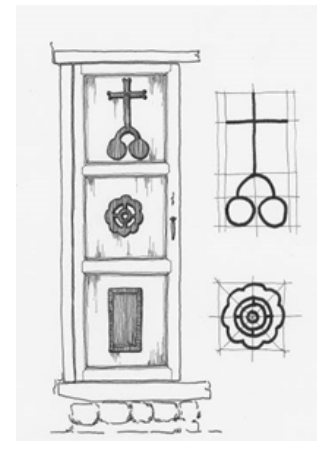

Figura 27: VALOR ESTÉTICO/ HABITABILIDAD Elementos decorativos simbólicos que al ingreso de una vivienda expresan identificación, protección y "gusto" de las personas que lo habitan (Solano, Cañar). Fuente: AVAC (2017). 


\section{Conclusiones}

A manera de reflexión final y conclusión, es importante destacar la función del dibujo como dato cualitativo muy importante para entender el espacio, la construcción y las formas de habitar en una edificación vernácula, de una manera integral que es capaz de trasmitir también emoción y nuevas percepciones de la vida.

Frente a los análisis cuantitativos, en este mismo caso de estudio (número de viviendas, repeticiones, tipologías, rasgos, porcentajes de permanencia y sustituciones), que han sido muy valiosos en la investigación previa que sustenta este artículo, es importante destacar la fuerza de simbolismo, expresión y comunicación emotiva que tiene el dibujo artístico como dato cualitativo.

Los datos cualitativos manejados con una visión crítica, como en este caso el dibujo, al cual se ha analizado a la luz de los datos cuantitativos, permiten una visión ampliada de contexto, vincula otras variables y posibilita una lectura amplia y precisa.

La experiencia de compartir los dibujos con un amplio público (maestros albañiles, campesinos, usuarios, estudiantes, profesionales), pone en evidencia que e dibujo como dato, como discurso, se difunde en un rango de cobertura más amplio, es capaz de sensibilizar, provocar emoción, reconocimiento, interés y actúa como fuente de conocimiento.

Recomendaciones: Se debe reforzar el dibujo en la enseñanza, para el registro, comunicación y valoración, suscitando así un aprendizaje de mayor detalle, profundización y apasionamiento. 


\section{Referencias bibliográficas}

- Cirvini, S. y Gómez, J. (2008). Los valores y significados del patrimonio vernáculo en tierra. Su relación con la conservación y construcción de nuevas obras en la región de Cuyo-Argentina. Mendoza, Argentina: Programa AHTER-Unidad y Territorio- INCIHUSA-CONICET..

- Correia, M. (2014). Versus. Lessons for vernacular heritage to sustainable architecture. Grenoble, Francia: CRAterre-ENSAG. Recuperado de http://www.esg.pt/ versus/pdf/versus_booklet.pdf

- Costa J. (1998). La esquemática. Buenos Aires, Argentina: Paidós.

- Eco, U. (1986). La estructura ausente. Introducción a la semiótica. Barcelona, España: Lumen.

- García, G., Tamayo, J. y Malo, G. (2017). Valoración de la arquitectura vernácula de Azuay y Cañar. En Seminario Internacional de Arquitectura y Construcción en Tierra (SIACOT). (pp. 658-671). La Paz, Bolivia: FAADU-UMSA / PROTERRA.

- ICOMOS. (1999). Carta del Patrimonio Vernáculo Construido. Recuperado de http://www.icomos.org/ charters/vernacular_sp.pdf

- ICOMOS. (2011). The Paris Declaration on heritage as a driver of development, Adopted at Paris, UNESCO headquarters. París, Francia: ICOMOS. Recuperado de https://www.icomos.org/Paris2011/GA2011 Declaration_de_Paris_EN_20120109.pdf

- Iglesia, R.E.J. (2011). Habitar, Diseñar. Práctica del Diseño y Resolución de Problemas. Buenos Aires, Argentina: Nobuko.
- Pesántez, M. y González, I. (2011). Arquitectura tradicional en Azuay y Cañar. Técnicas, creencias, prácticas y saberes. Quito, Ecuador: Instituto Nacional de Patrimonio Cultural (INPC).

- Rapoport, A. (1969) Vivienda y Cultura. Barcelona, España: Ed. Gustavo Gili.

- Sosa Velásquez, M. (2012).¿Cómo entender el territorio? Programa Gestión Pública y Desarrollo Territorial. Ciudad de Guatemala, Guatemala: Cara Parens, Universidad Rafael Landívar.

- Supic, P. (1982). Vernacular architecture: A lesson of the past for the future. Energy and Buildings, 5 (1), 43-54 doi:10.1016/0378-7788(82)90027-5

- Tillería González, J. (2010). La arquitectura sin arquitectos, algunas reflexiones sobre arquitectura vernacular. Revista AUS, (8), 12-15. doi:10.4206/ AUS.2010n8-04

- UNESCO. (2003) Convención para la salvaguardia del patrimonio cultural inmaterial. Recuperado de http:// unesdoc.unesco.org/images/0013/001325/132540s.pdf

- Vasilachis I. (2006). Estrategias de investigación cualitativa. Barcelona, España: Gedisa.

- Zorrilla, H. (2015). El concepto arquitectura vernácula. Arquitectura de casas [Blog]. Buenos Aires, Argentina. Recuperado de http://ww.arquitecturadecasas.info/elconcepto-arquitectura-vernacula/.

- Zunzunegui, S. (2007). Pensar la imagen. Universidad del País Vasco, España: Cátedra. 